\title{
Lower-dimensional quantum structures by selective growth and gas-phase nucleation
}

\author{
Kerry J. Vahala, Winston A. Saunders, Charles S. Tsai, Peter C. Sercel, ${ }^{\text {a) }}$ Tom Kuech, ${ }^{\text {b) }}$ \\ Harry A. Atwater, and Richard C. Flagan \\ California Institute of Technology, Pasadena, California 91125
}

(Received 3 February 1993; accepted 1 April 1993)

\begin{abstract}
There is increasing interest in the potential application of quantum dots and quantum wires to various solid state devices. In this article, the physics of lower-dimensional quantum structures will be reviewed with emphasis on applications. In addition, two fabrication approaches under investigation at Caltech will be discussed. The first is based on selective nucleation of III-V semiconductors on a patterned host substrate and the second is gas-phase nucleation of III-V clusters. Recent results on formation of nanocrystal GaAs and silicon clusters in the gas phase are presented.
\end{abstract}

\section{LOWER-DIMENSIONAL QUANTUM STRUCTURES}

There is increasing interest in the potential application of lower dimensional quantum wells (quantum wires and quantum dots) to various solid state devices. Applications include enhancing the performance of existing devices (e.g., semiconductor lasers based on quantum dot active layers ${ }^{1,2}$ ), the creation of materials with large optical nonlinearities, ${ }^{3}$ as well as new concepts concerning the generation of synthetic dopants. ${ }^{4}$ In the quantum well hierarchy that has emerged over the last decade (i.e., quantum wells, wires, and dots), quantum dots are the extreme quasi zero-dimensional case. Quantum dots are, in a sense, large manmade atoms (100-200 $\AA$ in diameter). Quantum wires, on the other hand, are the electronic analog of optical waveguides. As is true in conventional quantum wells, quantum dots and wires are small enough so that the electronic wave function boundary conditions significantly alter their electronic density of states, giving them properties very different from a larger (i.e., bulk) piece of material having the same shape.

In this article we review the requirements imposed on quantum structure fabrication by certain applications, review attempts to date directed at quantum wire and dot fabrication, and finally discuss two very different fabrication approaches under investigation at Caltech.

\section{FABRICATION CONSIDERATIONS}

To realize these structures there are two important points that emerge and ultimately determine the difficulty of fabrication. The quantum dot will be used to illustrate.

First is the necessary small diameter for a roughly spherical quantum dot if it is to exhibit quantum size effects at room temperature. Second is the uniformity of dot diameters that must be achieved in a dense array of quantum dots. A detailed discussion of these issues appears in Ref. 5. The results will now be quickly summarized. A first order upper limit on quantum dot size is established by requiring that the deBroglie wavelength for carriers with

\footnotetext{
${ }^{a)}$ Present address: University of Oregon.

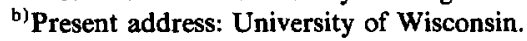

thermal energy $k T$ equal this size. If this condition is not realized then the discrete nature of the quantum dot electronic spectrum cannot be used to advantage at the operation temperature. It can also be shown that a lower limit on quantum dot size exists. ${ }^{5}$ These limits establish what has been described as the small quantum dot and the large quantum dot regimes and are illustrated in Fig. 1 for two material systems. For GaAs quantum dot material used at room temperature, it is apparent that a diameter of approximately $100 \AA$ is required.

Fabrication uniformity is the other key issue. Quantum dots are interesting individually, but must be fabricated in dense arrays to produce sufficient material for device applications. Unless the dots in these arrays are very uniform in size and shape (more specifically, uniform in quantum confinement energy), however, they lose their advantage over conventional material because the dispersion of dot diameters in an array leads to a dispersion or smearing of their combined electronic spectrum. In application to semiconductor laser active layers diameter dispersion-related smearing is exactly analogous to inhomogeneous broadening of the gain spectrum in gas laser systems. Its effect is to dilute the maximum attainable gain.

To illustrate the potential benefits of extreme fabrication uniformity, we have plotted in Fig. 2 the maximum attainable optical gain versus fabrication tolerance [essentially a root-mean-square (rms) deviation of the quantum dot radius averaged over the ensemble of quantum dots]. The tolerances assumed here are not attainable by any known fabrication approach, but will be revisited in a later section. The quantum dots are assumed to be GaAs (average diameter $100 \AA$ ) and the packing density is indicated. In addition to the fabrication tolerance (upper axis), an equivalent broadening rate is also used to label the plot. The cross-hatched region in the figure is beyond the geometrical packing limit of the quantum dots.

The plot shows that at a fixed dot density, the maximum attainable optical gain varies roughly inversely with fabrication tolerance. To understand the significance of this plot it necessary to realize that conventional semiconductor material when inverted to a density of slightly greater than $10^{18} \mathrm{~cm}^{-3}$ is capable of producing gains of $50-100 \mathrm{~cm}^{-1}$. 
SMALL QUANTUM DOT $\longrightarrow$ LARGE QUANTUM DOT

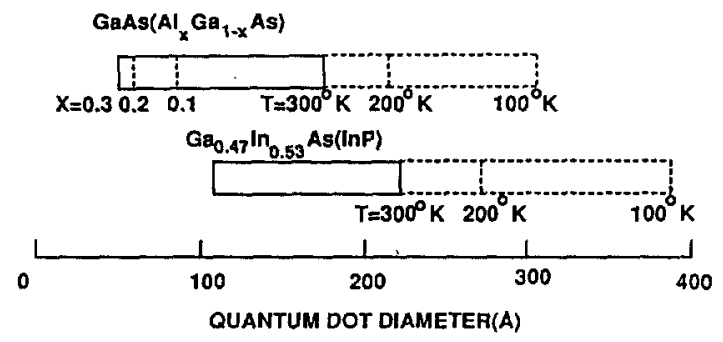

Fig. 1. Quantum dot size limits for various temperatures and material compositions in two different systems. The upper size limit is determined by the thermal deBroglie wavelength and the lower limit is determined by the existence of a critical radius below which there are no bound states (Ref. 5).

Figure 2 indicates that it is in principle possible to better this figure by several orders of magnitude. (Note: less stringent and hence more realistic tolerances also better these results. ${ }^{5}$ )

\section{APPROACHES TO QUANTUM STRUCTURE SYNTHESIS}

There are currently a large number of quantum structure fabrication techniques under investigation for fabrication of both quantum dots and quantum wires. The resulting structures fall very broadly into two classes: those that confine both carrier types and therefore lend themselves to both optical and carrier transport related devices, and those that lend themselves principally to carrier transport studies by confining only one carrier type. The earliest fabrication proposal falls into the latter category. It suggested using depletion in conjunction with a quantum well to realize a quantum wire structure for transport. ${ }^{6}$ The first attempt to fabricate wire structures utilized wet chemical etching of GaAs quantum well material combined with regrowth in molecular-beam epitaxy (MBE) to passivate

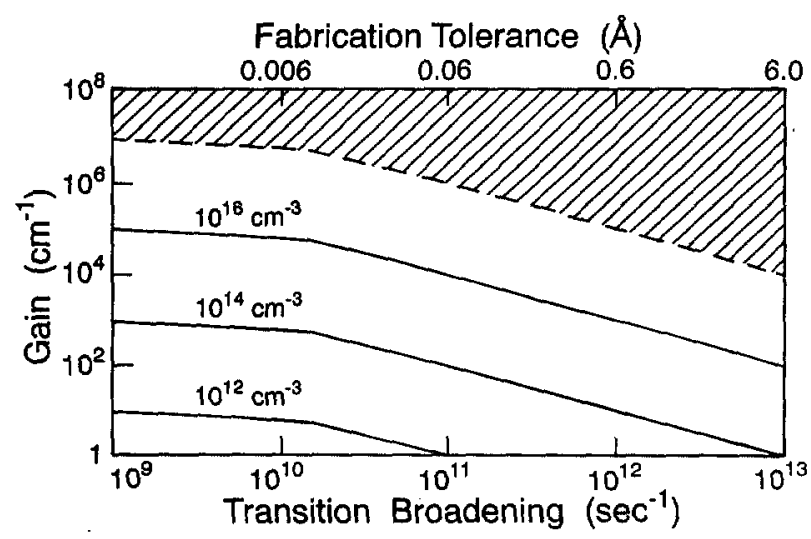

FIG. 2. Maximum attainable optical gain in an array of $100 \AA$ quantum dots characterized by the transition broadening rate (lower axis) or an equivalent fabrication tolerance (upper axis). The range of tolerances shown here are not attainable at present with any known fabrication approach; the plots are therefore intended to show what is possible, in principle. the surface. ${ }^{7}$ Although unsuccessful, the proposed structure should have confined both carrier types, and was intended to demonstrate quantum wire effects in the optical spectra. An early demonstration of quantum transport effects in wire structures actually occured much later and in metallic wires generated using electron beam lithography. A variety of interesting effects were observed related to the Aharonov-Bohm effect in ultrasmall current loops, ${ }^{8}$ the existence of universal conductance fluctuations in quantum wire systems at low temperature, ${ }^{9}$ and anomalies in the fractional quantum Hall effect at low temperatures. ${ }^{10}$

Several quantum wire and quantum dot fabrication techniques have been employed in semiconductor quantum well material with convincing results. One approach combines particle beam pattern generation with anisotropic etching techniques, such as reactive ion etching, to define both wire and dot structures. The resulting confinement has been used successfully by several groups to study the effects of reduced dimensionality on electronic transport $^{11,12}$ and optical properties. ${ }^{13,14}$ Wet chemical etching has also been used to produce similar structures. ${ }^{15}$ This has the advantage over reactive ion etching of minimizing damage to the semiconductor during etching. ${ }^{16}$ In addition, techniques based on impurity-induced disordering of quantum wells have been used to create both quantum wire and quantum dot structures that exhibit certain basic quantum size effects. ${ }^{17,18}$ Impurity induced disordering has also been combined with ion beam lithography to achieve simultaneous nanometer-scale pattern generation and impurity implanting. ${ }^{19,20}$ All of these approaches, however, suffer from interface defects which tend to degrade quantum structure optical efficiency. In addition, none of these approaches can reliably produce an abrupt quantumstructure interface.

More recently, a new class of techniques has emerged that directly address the interface issue. These approaches seek to fabricate a quantum structure and embed it in a host in a single growth step. One example of such an approach is growth on vicinal substrates ${ }^{21,22}$ which has been applied to generate quantum wire structures. ${ }^{23}$ This novel approach has the advantage of producing nanostructures without the need for any nanofabrication equipment (aside from a MBE reactor). Selective epitaxial growth of quantum structures, as discussed below, is another approach. ${ }^{24}$ Probably the most successful quantum wire fabrication approach demonstrated to date is growth on patterned substrates, first demonstrated by the Bellcore group. ${ }^{25}$ Lasing action from these structures has been realized although the wires fabricated to date are still not of sufficient quality to improve device performance over that obtainable with conventional quantum-well active layers.

In addition to quantum dot fabrication techniques that utilize substrates, there are a number of approaches directed towards cluster synthesis of quantum dots. Previously reported colloid-based syntheses of nanometer-scale GaAs clusters have represented important steps forward. Alivisatos et al. and Uchida et al. have synthesized crystalline GaAs clusters in quinoline solution which appear crystalline in transmission electron micrographs 
(TEM) ${ }^{26,27}$ However, molecular species from the synthesis itself mask the optical properties of the GaAs clusters.

In an alternate approach, Sandroff et al. ${ }^{28}$ using MBE, have grown nanometer scale GaAs clusters on silica substrates. This method produces stoichiometric and crystalline particles. However, particle shapes and sizes are highly nonuniform, and they are in intimate contact with the substrate.

\section{SELECTIVE GROWTH OF NANOSTRUCTURES}

In recent years organometallic chemical vapor deposition (OMCVD) has progressed to become an important process in the growth of high quality epitaxial layers of various semiconductor materials. Refinement of this technique has given it the ability to produce semiconductor material quality as pure or purer than other competitive growth techniques. Growth of monolayer abrupt interfaces, important for quantum device work, has also been demonstrated. A major advantage of this growth method over other competitive techniques such as MBE is that large areas and multiple wafers can be grown with high uniformity, making OMCVD ideally suited for large-scale production applications.

The subsequent lateral processing of these growths in the production of small scale optical devices is still a subject of intense research. Lateral current, carrier, and optical confinement can be obtained by processes such as etching and impurity-induced disordering. These processes all have shortcomings in the submicron regime. Exposed etched surfaces, while not important for large devices (where the surface to volume ratio is smaller) can be detrimental to nanometer scale devices due to surface states or etch damage. Impurity-induced disordering maintains a crystal-crystal interface thereby alleviating this problem but still suffers from a large interfacial region and introduces a high concentration of impurities.

Selective epitaxy may offer a means of circumventing many of the problems of lateral patterning. Selective epitaxy refers to the lateral spatially controlled growth of an epitaxial material within openings of a mask material residing on the substrate during growth. The masking material that we use is a dielectric layer of $\mathrm{Si}_{3} \mathrm{~N}_{4}$. Recent optimization of growth conditions and selection of growth precursors in the OMCVD growth on masked substrates have overcome the initial problems of selective growth. Suppression of polycrystalline growth on the masked regions as well as the high quality growth of $\mathrm{GaAs}$ in openings down to the micron size scale has been demonstrated. ${ }^{29}$ The selective area growth was found to be independent of feature size and geometry of the mask opening as well as the ratio of mask to open areas for this size regime.

We believe there are exciting possibilities in extending this procedure to device fabrication on the submicron and nanometer size scales. We will now present results we have obtained showing successful growth of GaAs dots and wires on this size scale. Some of these results appear in Refs. 24 and 30.

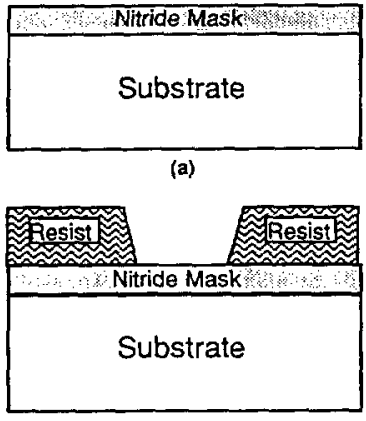

(b)

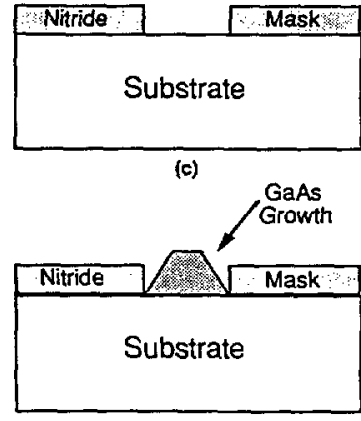

(d)
FIG. 3. Steps involved in the selective epitaxial growth of nanostructures.

The substrates used here were semi-insulating GaAs. A silicon nitride layer approximately $200 \AA$ thick was then grown on the sample by plasma enhanced chemical vapor deposition. Figure 3 depicts a summary of our fabrication steps.

In order to pattern the sample on a nanometer scale, a lithographic system capable of such resolution is essential. To achieve this we have modified the scanning electron microscope in our lab to do electron beam lithography. With this system we have achieved lines and dots with widths and diameters, respectively, of less than $500 \AA$. In this process an electron resist is deposited on the sample and a pattern is written into this resist using an electron beam. Developing the resist opens up the areas exposed by the electron beam. To transfer the resist pattern to the substrate the exposed nitride is etched away in a plasma etching system. The anisotropic nature of this etching system allows the resist pattern to be replicated in the nitride.

Specifics of the growth and sample preparation are described in Refs. 24, 29, and 30. A striking example of the faceted nature of the growth is beautifully demonstrated by the selective growth of dots. Figure 4 shows arrays of dots grown at $700{ }^{\circ} \mathrm{C}$. One can see the slow growth planes determining edge facets. These planes consist of the (110) type and the (111) type. We find that the uniformity of

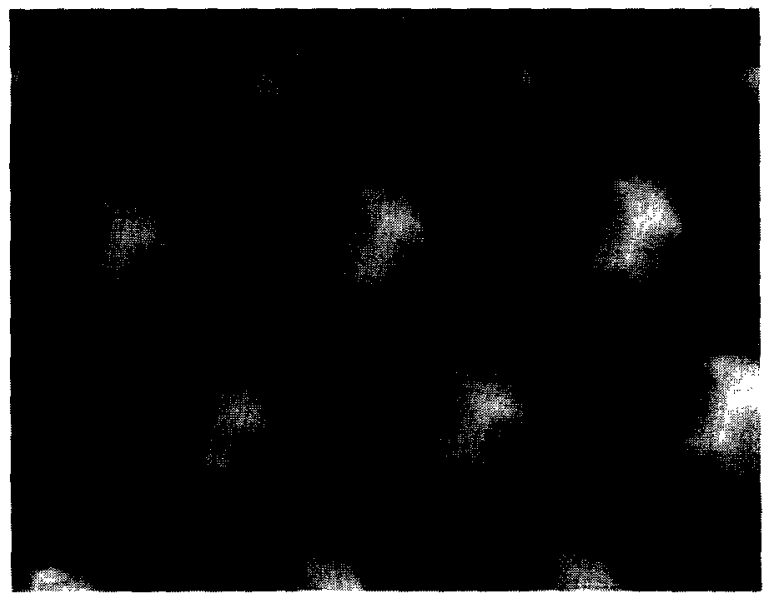

FIG. 4. An array of selectively grown GaAs dotlike structures. 


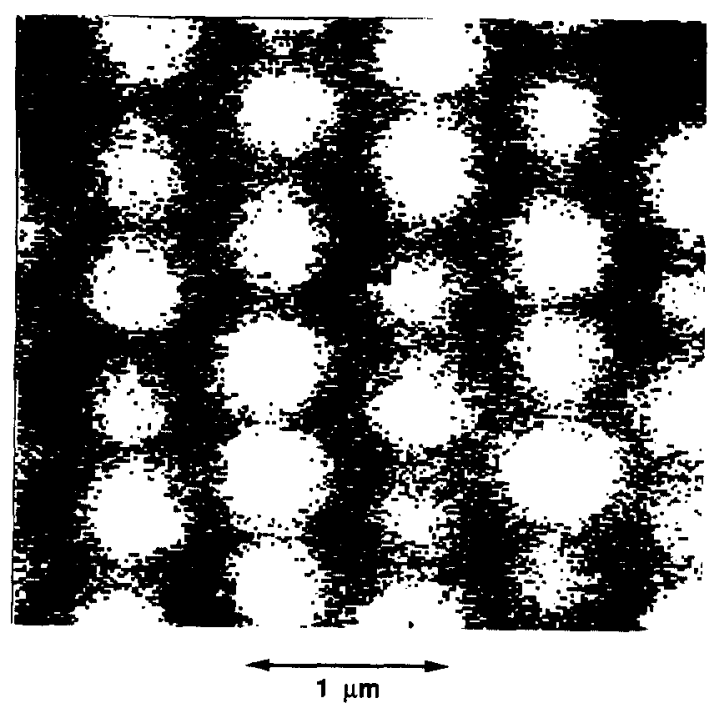

FIG. 5. Spectrally resolved cathodoluminescence from an array of selectively grown $1000 \AA \mathrm{GaAs}$ dots that have been overgrown and passivated by AlGaAs.

these dot arrays is very high, an important prerequisite for quantum devices. Again we note that growth of dots showing this structure were achieved down to our lowest diameter of $1000 \AA$ and smallest dot spacing of $4000 \AA$. In later work we were able to achieve selective growth in openings as small as $600 \AA$.

A second important requirement for utilization of this process for devices is the ability to grow AlGaAs over the GaAs for passivation and confinement. We have achieved overgrowth and passivation of both GaAs wire and dot arrays. To study these passivated structures we utilize a cathodoluminescence system. Cathodoluminescence (CL) is a very useful tool with which to study the luminescent properties of the selectively grown material as well as band gap modulation on a small scale. A spectrally resolved CL image is generated by rastering a scanning electron microscopy (SEM) electron beam in a selected area of the sample and detecting light at a specified wavelength. Figure 5 illustrates luminescence from an array of selectively grown $1000 \AA$ dots that have been overgrown by AlGaAs. In future work we plan to embed dot or wire regions between $p$ - and $n$-type layers thereby facilitating current injection. In such structures, the nitride layer could serve the additional purpose of acting as a current focusing layer to improve the injection efficiency into the quantum structures.

In addition to studying the selective nucleation process as a means to generation of nanostructures, we have also demonstrated that the highly faceted nature of selectively grown structures offers another way to produce quantum structures. In particular, wire doublet formation has been demonstrated using a technique we call facet modulation selective epitaxy. ${ }^{30}$ In addition, the variation of growth rate on different facets of a locally growing structure can be used to form pockets of low band gap material in the shape of a wire or a dot.

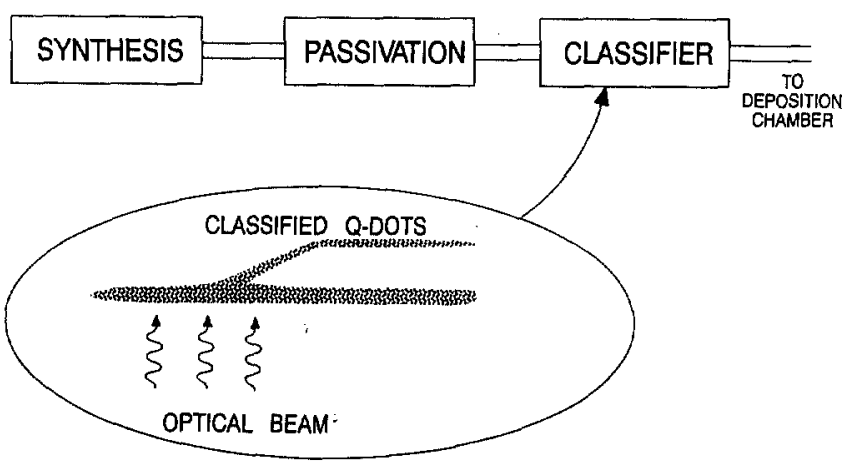

FIG. 6. Aerosol process for fabrication of monosized quantum dots.

\section{AEROSOL SYNTHESIS AND OPTICAL CLASSIFICATION OF QUANTUM DOTS}

We have previously proposed a method for cluster synthesis and classification that specifically addresses the issue of quantum dot uniformity. ${ }^{31}$ In this approach quantum dots would be actively classified according to the quantum dot transition energy of interest. The proposed quantum dot reactor would be configured in stages so that quantum dots would first be nucleated into small clusters, then passivated by growth of a layer of lattice matched material on the surface (e.g., GaAs dots with an AlGaAs passivation layer), and finally, sorted according to optical transition energy. Ultimately, the final stage of the reactor would supply classified optically active quantum-dot clusters to other systems. This could include a MBE system in which cluster beam deposition would embed the dots in an epitaxial layer, or alternatively, a system that embeds the dots in a dissimilar host for optical pumping purposes (e.g., the core of an optical fiber). The proposed process is illustrated in Fig. 6. The key feature of this process is the classification step which targets the actual quantum dot optical transition energy as the selection criterion. Indeed, in optical classification, size is not necessarily the relevant parameter since shape also affects the structure of the electronic spectrum: it is possible to imagine two quantum dots with different sizes and shapes, but which have identical ground state quantum confinement energies.

Even apart from their physical properties, which depart from those of bulk electronic materials, the realization of quantum dots as an array of passivated clusters as discussed below has interesting technological implications, since they can potentially be integrated into any optical medium, such as a thin film active region, waveguide or fiber, without regard to the electronic quality of the dothost interface.

The classification mechanism utilizes laser radiation resonant with the prescribed quantum dot transition. Quantum dots resonant with the laser are deflected via photon momentum transfer and are thereby classified. The process is very much like that used in certain laser isotope separation schemes. ${ }^{32}$ Provided that single frequency lasers are used, the classified dots can have an optical emission spectrum which is, in principle, no wider than the homoge- 
neous line shape function of the individual quantum dots. Photon momentum pressures are easily sufficient to deflect dots in vacuum. Alternatively, dots could be tagged by photoionization and subsequently removed electrostatically.

As an important first step towards this goal we have demonstrated a new vapor phase synthesis approach for GaAs clusters. ${ }^{33}$ Vapor phase cluster synthesis has several advantages over other techniques, including controlled chemical purity and the generation of clusters with free surfaces. However, it also presents considerable technical challenges. At low growth temperatures, atomic mobilities are low in the solid phase, inhibiting formation of crystalline clusters. At high growth temperatures, however, maintaining stoichiometry is difficult owing to widely different vapor pressures of the elemental sources (in this case arsenic and gallium).

\section{VAPOR-PHASE SYNTHESIS OF III-V SEMICONDUCTOR CLUSTERS}

\section{A. Organometallic vapor phase epitaxy (OMVPE) growth of clusters}

We have recently reported the first direct observation of gas-phase homogeneous nucleation of nanometer-scale GaAs clusters in an atmospheric OMVPE reactor. ${ }^{33}$ In addition to shedding light on a fundamental question about the reaction kinetics involved in OMVPE, this is the first report of gas-phase synthesis of nanometer-scale III/V clusters from volatile precursor molecules. We have determined conditions under which nearly defect free, $10-20 \mathrm{~nm}$ scale $\mathrm{GaAs}$ microcrystallites form. These particles are in the quantum size regime which suggests that the process may be used to form optically active quantum dots. Thus gas-phase homogeneous nucleation in OMVPE, which has been regarded until now as a troublesome parasitic reaction, may ultimately form the basis of an aerosol technology for the fabrication of novel optoelectronic devices.

A TEM micrograph of the collected deposits from the OMVPE reaction reveals it to consist of individual GaAs microcrystallites and some chainlike agglomerates (Fig. 7). The electron diffraction pattern of the particles, shown in the inset of Fig. 7, indexes to cubic zinc blende structure. Figure 8 shows a high-resolution transmission electron micrograph (HRTEM) of an agglomerate of smaller primary particles. In practice, HRTEM is easier to perform on the agglomerated clusters than on individual particles, owing to the fact that the agglomerates are better able to dissipate excess charge and heat induced by the electron beam than are individual particles. Within several of the clusters shown, lattice fringes and faceting indicative of $\langle 111\rangle$ bounding planes are clearly evident. Some of the crystalline faces have as few as 30 atomic rows. The lattice fringes on the particles extend to within $1 \mathrm{~nm}$ of the cluster surface, consistent with the formation of an amorphous oxide layer on the particles on exposure to air. Data on cluster size distribution as a function of growth temperature are presented in Fig. 9. The data are fitted by log-normal distri-

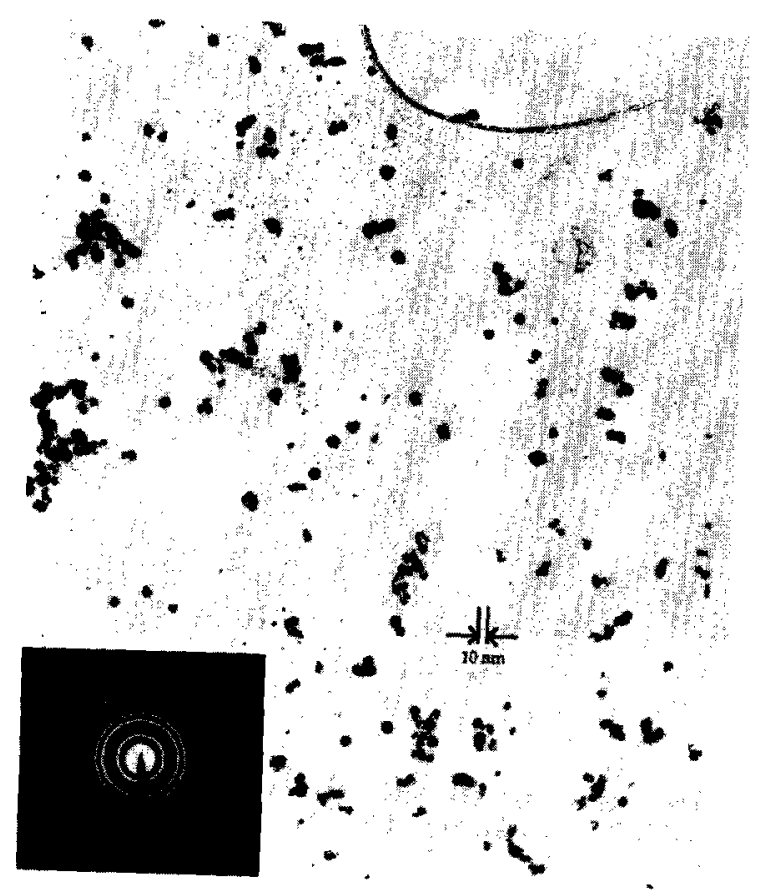

FIG. 7. Bright field TEM micrograph of GaAs clusters collected on a holey carbon substrate. Inset: electron diffraction pattern of the clusters.
The pattern indexes to zinc blende.

butions having mean diameters in the 100-200 $\AA$ size range.

We have also developed another approach for III-V cluster synthesis. This technique is based on discharge of a short, intense electrical current through a conducting III-V wire in an inert ambient gas. Subsequent cooling of

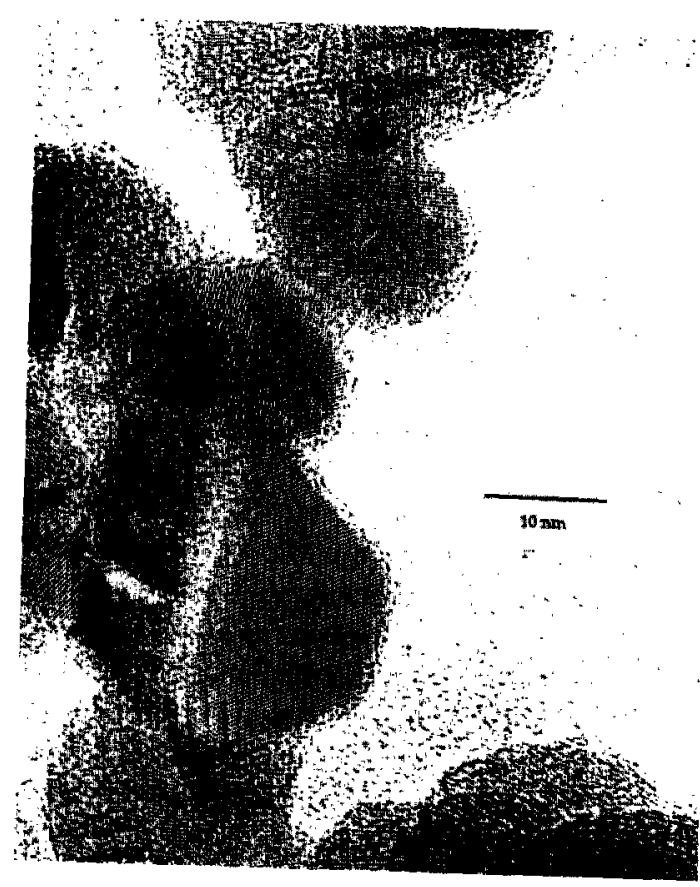

FIG. 8. HRTEM micrograph of a chain agglomerate produced under the same conditions as Fig. 7 . 

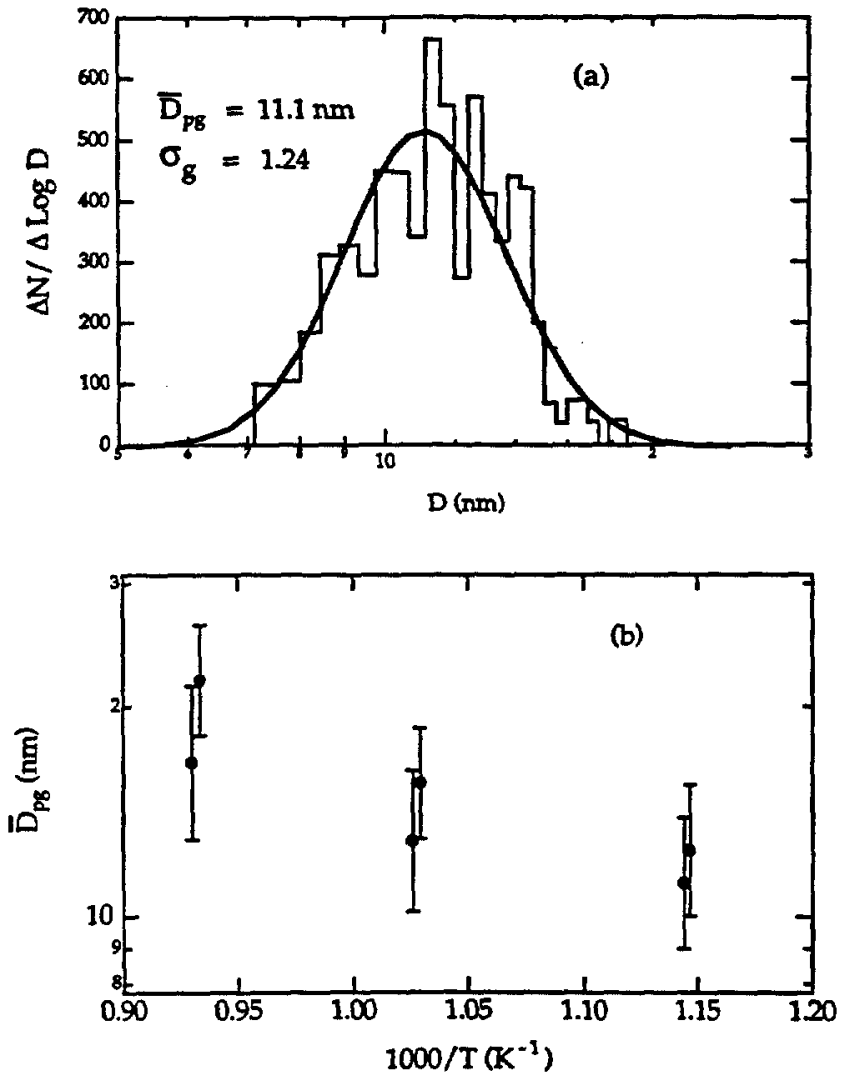

FIG. 9. Analysis of size distribution of GaAs clusters produced at various temperatures. (a) Representative data for a run with growth temperature $600^{\circ} \mathrm{C}$. The solid line is a log-normal fit. (b) Semilog plot of median particle diameter vs reciprocal temperature. The geometric standard deviation for each point is also shown.

the hot vapor produced in this process leads to formation of stoichiometric III-V crystals in the $10 \mathrm{~nm}$ size regime. The reader is referred to Ref. 34 for further details.

\section{B. Cluster surface passivation}

In order to produce clusters with desirable optical properties, such as high photoluminescence efficiency and large nonradiative carrier lifetimes, it is necessary to eliminate electrically active defects within GaAs clusters. Most critical is the need to eliminate surface states which produce deep levels within the energy gap of the dot, and reduce the nonradiative carrier lifetime. The most straightforward

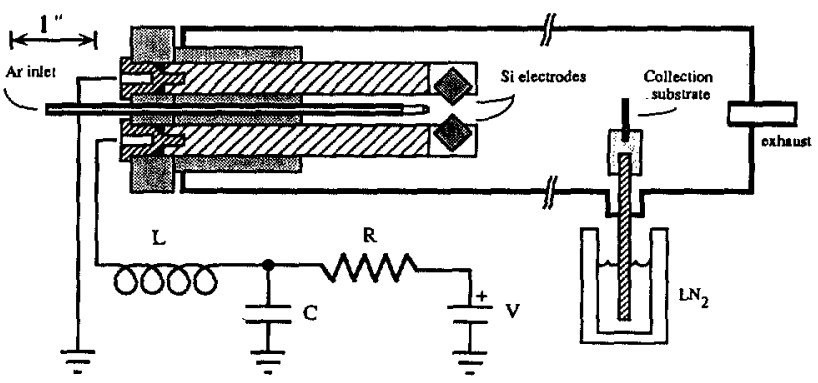

FIG. 10. Schematic of silicon cluster reactor.
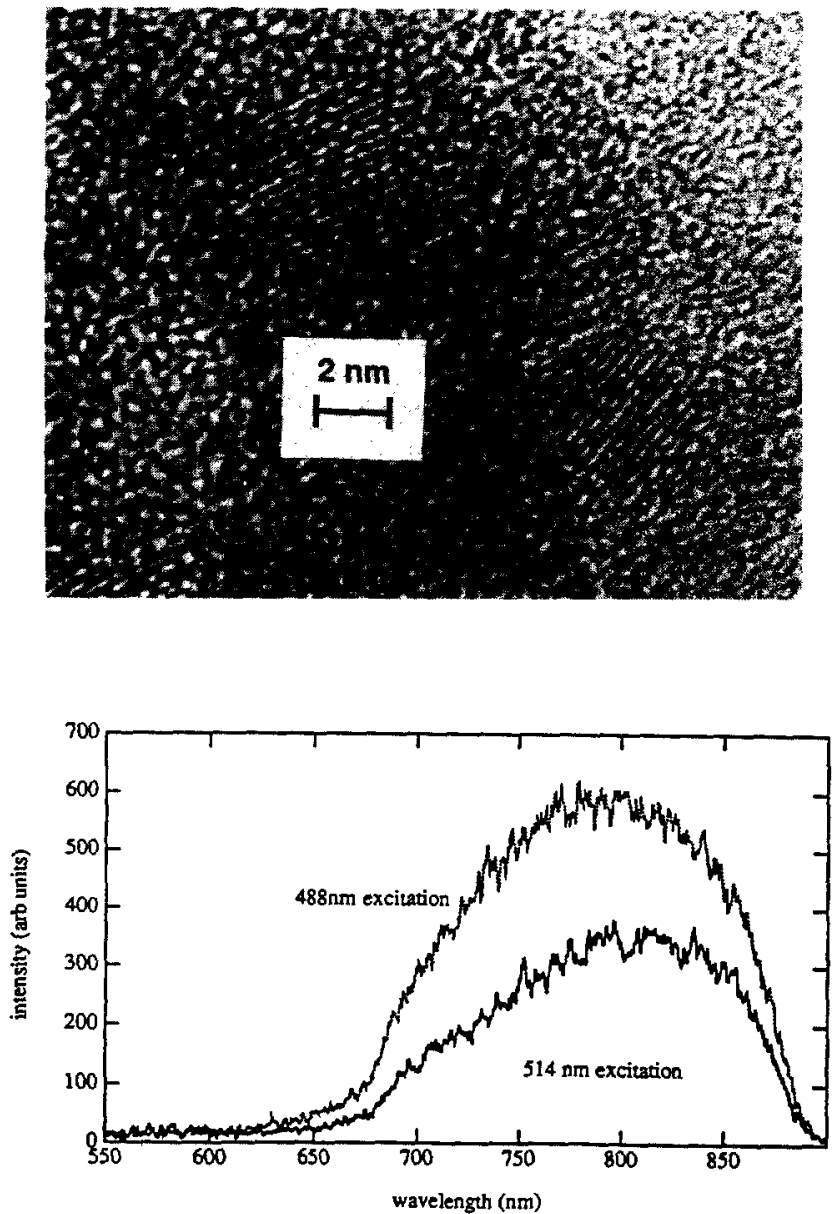

FIG. 11. HRTEM images and luminescence spectra of silicon material formed in the reactor of Fig. 10.

method for elimination of defect states associated with $\mathrm{GaAs}$ surfaces is to encapsulate each dot within a semiconductor of wider energy gap, and to insure that the density of electronic interface states is low. The most appropriate wide gap material is AlGaAs. Indeed, the existence of a well-developed GaAs-AlGaAs heterojunction technology was the primary motivation for the choice of GaAs as the material system-and OMVPE as the growth technique-to be studied. We are currently investigating the overgrowth of GaAs clusters by AlGaAs.

\section{VAPOR PHASE SYNTHESIS OF NANOCRYSTALLINE SILICON}

In recent years there has been much excitement surrounding the discovery of efficient luminescence from porous silicon generated by chemical etching. We have recently discovered and reported on a new route to synthesis of optical active silicon. ${ }^{35}$ A schematic drawing of our reactor is illustrated in Fig. 10. In it, two silicon electrodes are vaporized by a pulsed electrical discharge in the presence of an argon flow. The carrier gas cools the silicon vapor produced by the spark, producing a condensate that is subsequently deposited on a collection grid down stream in the process tube. 
The collected material has been analyzed by HRTEM and is found to contain nanocrystals of silicon in the 2-4 $\mathrm{nm}$ size regime. This material luminesces with a spectrum similar to that seen in porous silicon samples. ${ }^{36}$ Both HRTEM images and luminescence spectra are provided in Fig. 11. Although further data are required to define precisely the mechanisms by which this material can produce visible luminescence, the preliminary evidence is consistent with an explanation based on quantum confinement of the electron-hole pair.

\section{SUMMARY AND CONCLUSIONS}

In this article, we have reviewed several critical issues involved in fabrication of nanometer scale structures called quantum wires and quantum dots. In addition, we have presented several routes toward the synthesis of nanometer-scale structures in compound and elemental semiconductors. It is evident that normal semiconductor fabrication techniques are not suitable for the production of precisely controlled two and three-dimensional features at the nanometer length scale. New techniques, such as those discussed above and elsewhere, are needed to take advantage of the enhancements in performance expected from quantum confinement effects in nanometer-scale semiconductors.

'Y. Arakawa, K. Vahala, and A. Yariv, Appl. Phys. Lett. 45, 950 (1984).

${ }^{2}$ K. Vahala, Y. Arakawa, and A. Yariv, Appl. Phys. Lett. 50, 365 (1987).

${ }^{3}$ E. Hanamura, Phys. Rev. B 37, 1273 (1988).

${ }^{4}$ P. Sercel and K. Vahala, Appl. Phys. Lett. 57, 1569 (1990).

${ }^{5}$ K. Vahala, J. Quantum Electron. QE-24, 523 (1988).

${ }^{6}$ H. Sakaki, Jpn. J. Appl. Phys. 19, L735 (1980).

${ }^{7}$ P. M. Petroff, A. C. Gossard, R. A. Logan, and W. Wiegmann, Appl. Phys. Lett. 41, 635 (1982).

${ }^{8}$ R. Webb, S. Washburn, C. Umbach, and R. Laibowitz, Phys. Rev. Lett. 54, 2696 (1985).

${ }^{9}$ W. J. Skocpol, P. M. Mankiewich, R. E. Howard, L D. Jackel, D. M.

Tennant, and A. D. Stone, Phys. Rev. Lett. 56, 2865 (1986).

${ }^{10}$ M. L. Roukes, A. Scherer, S. J. Allen, Jr., H. G. Craighead, R. M. Ruthen, E. D. Beebe, and J. P. Harbison, Phys. Rev. Lett. 59, 3011 (1987).

${ }^{11}$ M. A. Reed, J. N. Randall, R. J. Aggarwal, R. J. Matyi, T. M. Moore, and A. W. Wetsel, Phys. Rev. Lett. 60, 535 (1988).

${ }^{12}$ S. Thoms, I. McIntyre, S. P. Beaumont, M. Al-Mudares, R. Cheung, and C. D. W. Wilkenson, J. Vac. Sci. Technol. B 6, 127 (1988).

${ }^{13}$ K. Kash, A. Scherer, J. M. Worlock, H. G. Craighead, and M. C. Tamargo, Appl. Phys. Lett. 49, 1043 (1986).

${ }^{14}$ H. Temkin, G. J. Dolan, M. B. Panish, and S. N. G. Chu, Appl. Phys. Lett. 50, 413 (1987).

${ }^{15}$ Y. Miyamoto, M. Cao, Y. Shingai, K. Furuya, Y. Suematsu, K. G. Ravikumar, and S. Arai, Jpn. J. Appl. Phys. 26, L225 (1987).

${ }^{16}$ A. Scherer, H. G. Craighead, M. L. Roukes, and J. P. Harbison, J. Vac. Sci. Technol. B 6, 227 (1988).

${ }^{17}$ J. Cibert, P. M. Petroff, G. J. Dolan, S. J. Pearton, A. C. Gossard, and J. H. English, Appl. Phys. Lett. 49, 1275 (1986).

${ }^{18}$ H. A. Zarem, P. C. Sercel, M. E. Hoenk, A. Yariv, and K. J. Vahala, Paper TUAS at the 1989 Conference on Quantum Wells for Optics and Optoelectronics, Salt Lake City (unpublished).

${ }^{19}$ T. Hiramoto, K. Hirakawa, and T. Ikoma, J. Vac. Sci Technol. B 6, 1014 (1988).

${ }^{20}$ R. L. Kubena, R. J. Joyce, J. W. Ward, H. L. Garvin, F. P. Stratton, and R. G. Brault, J. Vac. Sci. Technol. B 6, 353 (1988).

${ }^{21}$ J. M. Gaines, P. M. Petroff, H. Kroemer, R. J. Simes, R. S. Geels, and J. H. English, J. Vac. Sci. Technol. B 6, 1378 (1988).

${ }^{22}$ T. Fukui and H. Saito, Appl. Phys. Lett. 50, 824 (1987).

${ }^{23}$ M. Tsuchiya, J. M. Gaines, R. H. Yan, R. J. Simes, P. O. Holtz, L. A. Coldren, and P. M. Petroff, Phys. Rev. Lett. 62, 466 (1989).

${ }^{24}$ J. Lebens, C. S. Tsai, K. J. Vahala, and T. F. Kuech, Appl. Phys. Lett. 56, 2642 (1990).

${ }^{25}$ E. Kapon, D. M. Hwang, and R. Bhat, Phys. Rev. Lett. 63, 430 (1989).

${ }^{26}$ M. A. Olshavsky, A. N. Goldstein, and A. P. Alivisatos, J. Am. Chem. Soc. 112, 9438 (1990).

${ }^{27}$ H. Uchida, C. J. Curtis, and A. J. Nozik, J. Phys. Chem. 95, 5382 (1991).

${ }^{28}$ C. J. Sandroff, J. P. Harbison, R. Ramesh, M. J. Andejco, M. S. Hedge, D. M. Hwang, C. C. Chang, and E. M. Vogel, Science 245, 391 (1989).

${ }^{29}$ T. F. Kuech, M. A. Tischler, and R. Potemski, Appl. Phys. Lett. 54 910 (1989).

${ }^{30}$ C. S. Tsai, J. A. Lebens, C. C. Ahn, A. Nouhi, and K. J. Vahala, Appl. Phys. Lett. 60, 240 (1992).

${ }^{31}$ K. Vahala, H. Atwater, R. Flagan, C. Tsai, J. Lebens, P. C. Sercel, and W. Saunders, Quantum Electronics and Laser Science 1991, Baltimore, Invited Paper JThC1 (unpublished).

${ }^{32}$ Laser Photochemistry, Tunable Lasers, and Other Topics, edited by S. F. Jacobs, M. Sargent III, M. O. Scully, and C. T. Walker (AddisonWesley, Reading, MA, 1976).

${ }^{33}$ P. C. Sercel, W. A. Saunders, H. A. Atwater, K. J. Vahala, and R. C. Flagan, Appl. Phys. Lett. 61, 696 (1992).

${ }^{34}$ W. A. Saunders, P. C. Sercel, H. A. Atwater, K. J. Vahala, and R. C. Flagan, Appl. Phys. Lett. 60, 950 (1992).

${ }^{35}$ W. A. Saunders, K. J. Vahala, H. A. Atwater, R. C. Flagan, and P. C. Sercel, Proceedings of the 1992 Fall Materials Research Society Meeting, 30 November -4 December, Boston, MA [Proc. Mater. Res. Soc. (to be published)], paper F14.13.

${ }^{36}$ L. T. Canham, Appl. Phys. Lett. 57, 1046 (1990). 